\title{
Aortic Aneurysm-associated Disseminated Intravascular Coagulation that Responded Well to a Switch from Warfarin to Rivaroxaban
}

\author{
Yasuko Kadohira, Shinya Yamada, Erika Matsuura, Tomoe Hayashi, \\ Eriko Morishita, Shinji Nakao and Hidesaku Asakura
}

\begin{abstract}
:
We describe a case in which uncontrolled chronic disseminated intravascular coagulation (DIC) caused by an aortic aneurysm that was exacerbated by chemotherapy for lung cancer, showed dramatic improvement when warfarin, which was being administered for atrial fibrillation, was replaced by rivaroxaban, a direct oral anticoagulant (DOAC). The present case is interesting because a DOAC was effective in treating DIC due to an aortic aneurysm, whereas warfarin, another oral anticoagulant, was ineffective. In controlling DIC, it is important to inhibit activated coagulation factors such as thrombin and activated factor $\mathrm{X}$, rather than the coagulation factors, which act as substrates.
\end{abstract}

Key words: disseminated intravascular coagulation, aortic aneurysm, fibrinolytic activation, direct oral anticoagulant, rivaroxaban

(Intern Med 56: 2913-2917, 2017)

(DOI: 10.2169/internalmedicine.8666-16)

\section{Introduction}

Disseminated intravascular coagulation (DIC) is a pathological condition in which systemic, persistent, and marked coagulation activation in the presence of an underlying disease and varying degrees of fibrinolytic activation are seen simultaneously. DIC can be accompanied by organ damage due to microcirculatory disorders, and by bleeding symptoms due to consumption coagulopathy and marked fibrinolytic activation (1).

DIC associated with aortic aneurysms often follows a long course and becomes chronic DIC (2). Even when clear coagulation-fibrinolytic activation is present, the patient may show no symptoms and DIC may go unnoticed. On the other hand, DIC is sometimes diagnosed when trauma or an invasive procedure triggers sudden difficulty in achieving hemostasis, necessitating therapeutic intervention. Difficulty in treating aortic aneurysm is not uncommon, and such patients require long-term pharmacotherapy for DIC. In this scenario, the ideal treatment would only minimally detract from the patient's quality of life.

Although reducing the coagulation factors, which act as substrates, does not improve DIC (DIC can even develop in patients with fulminant hepatitis with depleted coagulation factors), it is necessary to reduce the activated coagulation factors to control DIC. Many patients with DIC have been treated with unfractionated heparin in order to inhibit the activated coagulation factors via antithrombin $(3,4)$. However, such therapies have a drawback, as the patient must be constrained for $24 \mathrm{~h}$ to receive continuous drip infusion. Thus, this approach is not suitable for the treatment of chronic DIC in an outpatient setting. Although the intravenous administration of danaparoid and the subcutaneous administration of heparin do not always constrain patients, the ability to perform this treatment in an outpatient setting is also limited. The self-administration of subcutaneous heparin has recently been approved for insurance coverage in Japan; however, self-administration is also a burden to patients. Accordingly, the ability to control DIC with an oral anticoagulant would be greatly beneficial to patients with chronic DIC.

The oral anticoagulants that are currently in clinical use 


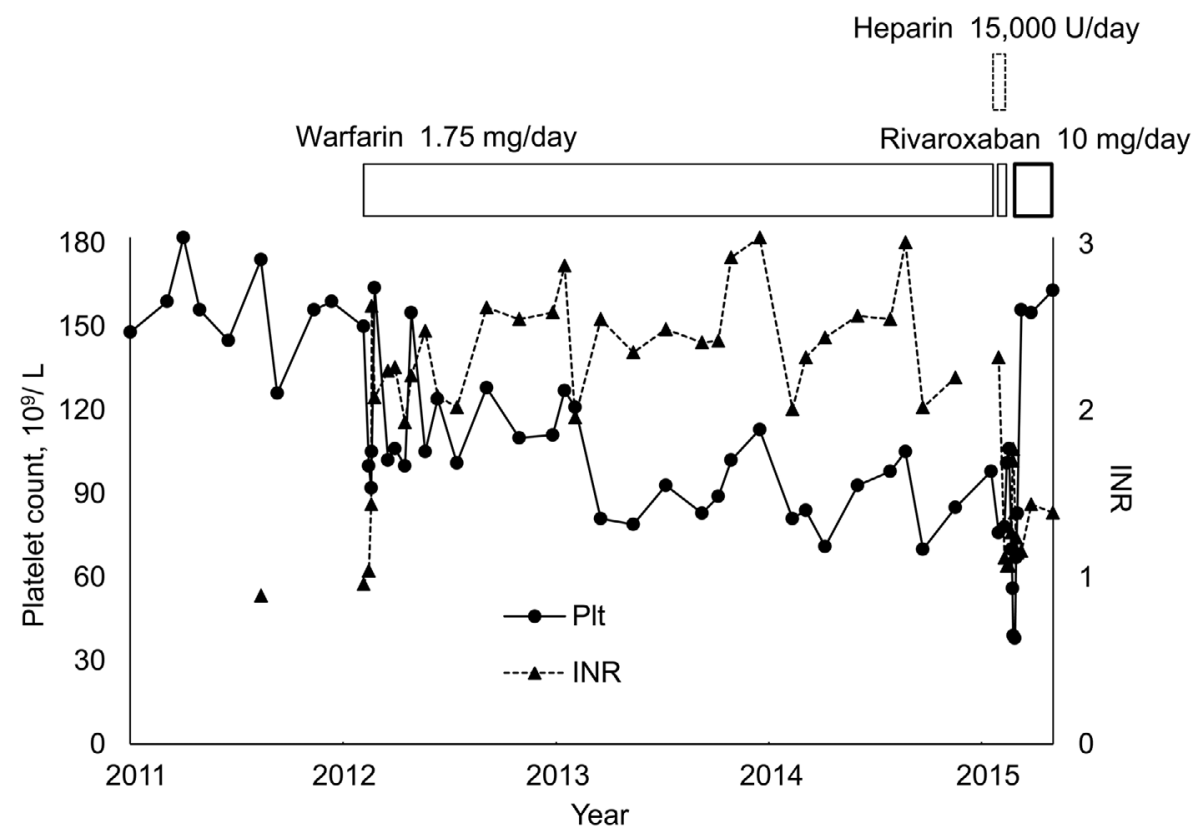

Figure 1. The changes in the platelet counts and fibrinogen levels during warfarin treatment.

include warfarin and direct oral anticoagulants (DOACs). DOACs inhibit activated coagulation factors such as thrombin and activated factor $\mathrm{X}$, and are used to prevent and treat non-valvular atrial fibrillation and deep venous thrombosis. The ability of DOACs to inhibit activated coagulation factors makes them fundamentally different from warfarin, which reduces the activity of vitamin K-dependent coagulation factors as substrates and proenzymes. Because activated protein $\mathrm{C}$ can improve coagulation abnormalities, warfarin can worsen coagulopathy by reducing the expression of protein $\mathrm{C}$ and protein $\mathrm{S}$ (a cofactor of protein $\mathrm{C}$ ).

In theory, DOACs should be effective for treating DIC, since they inhibit activated coagulation factors. In fact, we have already reported that the DOAC rivaroxaban was effective for treating chronic DIC associated with aortic aneurysms (5). On the other hand, warfarin, another oral anticoagulant, would be thought to be ineffective for DIC, since it does not show inhibitory activity against activated coagulation factors and it inhibits the generation of protein $\mathrm{C}$ and protein $\mathrm{S}$. Nevertheless, warfarin has been expected to show some degree of efficacy against DIC, perhaps because of its convenience as an oral drug, and it has been administered to DIC patients (6-10). Essentially, however, warfarin and DOACs are not indicated for the treatment of DIC.

We herein report the case in which the dramatic improvement and long-term control of DIC was achieved in a patient with chronic DIC caused by an aortic aneurysm that was uncontrolled by warfarin (administered for atrial fibrillation) when warfarin was switched to rivaroxaban. We reconfirmed that rivaroxaban, a DOAC, was effective when for treating chronic DIC caused by an aortic aneurysm. On that basis, it is considered that this demonstration - namely, that warfarin is ineffective against DIC, even in patients such as this one - is highly significant.

\section{Case Report}

The patient was a 69-year-old man who was first treated at our hospital for thoracoabdominal aortic aneurysm, angina, and hypertension in 2009. Paroxysmal atrial fibrillation manifested in 2010. In February 2012, he experienced a stroke immediately after the insertion of an aortic aneurysm stent graft, and the administration of warfarin was started to treat paroxysmal atrial fibrillation. His $\mathrm{CHA}_{2} \mathrm{DS}_{2}$-VASc score was 6 (CHADS $_{2}$ score was 4). From around 2013, his platelet count decreased to approximately $70-100 \times 10^{9} / \mathrm{L}$ (reference range: $130-350 \times 10^{9} / \mathrm{L}$ ), and chronic DIC associated with the aortic aneurysm was suspected (Fig. 1). During this time, warfarin showed a prothrombin time-international normalized ratio (PT-INR) of approximately 2.0-3.0 and the anticoagulation control was good; however, the patient's platelet count remained low and continued to gradually decline. In addition, the fibrin/fibrinogen degradation product (FDP) level during this time was $50-130 \mu \mathrm{g} / \mathrm{mL}$ (reference value: $>5.0 \mu \mathrm{g} / \mathrm{mL}$ ) while the D-dimer level was $30-50 \mu \mathrm{g} / \mathrm{mL}$ (reference value: $>1.0 \mu \mathrm{g} / \mathrm{mL}$ ). Fibrinogen (Fbg) showed large fluctuations, falling to approximately $140 \mathrm{mg} / \mathrm{dL}$ (reference range: $183-381 \mathrm{mg} / \mathrm{dL}$ ). Thoracoabdominal contrastenhanced computed tomography (CT) was performed in January 2015 in order to observe the course after thoracoabdominal aortic aneurysm surgery, and a nodule was noted in the right upper lobe. In February 2015, positron emission tomography (PET)-CT revealed multiple lymph node metastases, and the patient was admitted to our hospital. Squamous cell carcinoma was diagnosed based on the results of right cervical lymph node biopsy. Warfarin was replaced with heparin $(15,000 \mathrm{U} / 24 \mathrm{~h})$ for 19 days to allow lymph node biopsy (warfarin was administered before and after that in- 
terval).

Chemotherapy was started for lung cancer in March 2015. On day 2 of chemotherapy, the patient's platelet count was $56 \times 10^{9} / \mathrm{L}$; it decreased further to $39 \times 10^{9} / \mathrm{L}$ on day 3 of chemotherapy. A coagulation test on day 3 of chemotherapy showed the following results: PT, $20.1 \mathrm{~s}$ (reference range: 10.5-12.9 s); PT-INR (while on warfarin), 1.77; activated partial thromboplastin time (APTT), $40.9 \mathrm{~s}$ (reference range: 27.3-40.3 s); Fbg, >50 mg/dL; FDP, $139.7 \mu \mathrm{g} / \mathrm{mL}$; D-dimer, $64.4 \mu \mathrm{g} / \mathrm{mL}$; antithrombin, $106 \%$ (reference range: 70 $130 \%$ ); thrombin-antithrombin complex (TAT), $82.8 \mathrm{ng} / \mathrm{mL}$ (reference value: $>4 \mathrm{ng} / \mathrm{mL}$ ); plasmin- $\alpha_{2}$-antiplasmin inhibitor complex (PAP), $11.4 \mu \mathrm{g} / \mathrm{mL}$ (reference value: $>1.1 \mu \mathrm{g} /$ $\mathrm{mL}$ ); plasminogen, $68 \%$ (reference range: 70-130\%); and $\alpha_{2}$ antiplasmin, $49 \%$ (reference range: $70-130 \%$ ). These data indicated marked coagulation-fibrinolytic activation. The patient was diagnosed with an exacerbation of aortic aneurysm-associated enhanced-fibrinolytic-type DIC (according to diagnostic criteria for DIC with enhanced fibrinolysis) (11) in response to chemotherapy.

The risk of bleeding was considered to be high due to the advanced thrombocytopenia and marked fibrinolytic activation. Thus, warfarin was discontinued on day 3 of chemotherapy. However, as there was a risk of thrombosis due to atrial fibrillation, the oral administration of rivaroxaban (10 $\mathrm{mg} /$ day) was started on day 7 of chemotherapy (after confirming that PT-INR had decreased to 1.39). The patient had renal dysfunction, with a body weight of $63.0 \mathrm{~kg}$, a serum creatinine level of $1.97 \mathrm{mg} / \mathrm{dL}$ and a creatinine clearance of $32 \mathrm{~mL} / \mathrm{min}$. We therefore set the dose of rivaroxaban to 10 mg. On day 8 of chemotherapy, tests showed clear improvements in DIC (Fig. 2): platelet count, $67 \times 10^{9} / \mathrm{L}$; Fbg, 113 $\mathrm{mg} / \mathrm{dL}$; FDP, $98.0 \mu \mathrm{g} / \mathrm{mL}$; and D-dimer, $37.2 \mu \mathrm{g} / \mathrm{mL}$. The test results on day 10 of chemotherapy were as follows: platelet count, $83 \times 10^{9} / \mathrm{L}$; Fbg, $138 \mathrm{mg} / \mathrm{dL}$; FDP, $62.9 \mu \mathrm{g} /$ $\mathrm{mL}$; and D-dimer, $25.5 \mu \mathrm{g} / \mathrm{mL}$. Moreover, TAT, a coagulation activation marker, and PAP, a fibrinolytic activation marker, showed clear decreases after the start of rivaroxaban. No bleeding side effects accompanied the administration of rivaroxaban.

After completing the first course of chemotherapy, even though the patient's lung cancer was not reduced (stable disease), his DIC was improved by rivaroxaban, which was administered for atrial fibrillation. The control of DIC continued to be good, and the patient was not considered to be at risk of bleeding. For these reasons, the patient was discharged on day 23 of chemotherapy. After discharge, the patient's platelet count did not decrease due to DIC, and it was possible to administer chemotherapy once every 4 weeks on an outpatient basis. The patient's platelet count was maintained in the range of $150-200 \times 109 / \mathrm{L}$, while his Fbg level remained in the range of $200-300 \mathrm{mg} / \mathrm{dL}$ for 13 months after the start of rivaroxaban therapy, until the patient died of lung cancer.

\section{Discussion}

Various treatments have been reported to be effective for chronic DIC accompanied by the strong fibrinolytic activation that is associated with blood vessel-related diseases such as aortic aneurysm and giant hemangioma. Examples include nafamostat mesilate, heparin and recombinant thrombomodulin (12-14). However, those treatments are all administered by drip infusion, which makes them impractical for outpatient therapy.

The patient in this case had been newly diagnosed with lung cancer, but had a history of atrial fibrillation with cerebral infarction, and warfarin was being administered as anticoagulation therapy. The patient's DIC worsened immediately after the initiation of chemotherapy, due to the activation of the extrinsic coagulation pathway by the large amount of tissue factor that was released from tumor cells that had been disintegrated by chemotherapy and warfarin had to be discontinued due to the potential for bleeding symptoms. On the other hand, we wanted to continue anticoagulation therapy due to the patient's atrial fibrillation. We were the first to report that rivaroxaban - an oral activated factor X inhibitor - was effective for the treatment of DIC associated with aortic aneurysms (5). Subsequently, other authors have reported that rivaroxaban is effective for treating DIC associated with abdominal aortic aneurysms (15). Based on our earlier experience, in which a patient was treated with rivaroxaban (after we obtained approval from our hospital's medical ethics committee and after obtaining informed consent), following confirmatory reports from others, and after verifying that the PT-INR had been corrected following the discontinuation of warfarin, we initiated treatment with rivaroxaban. Although he was already hospitalized, we did not want to constrain the patient, who had a good general condition. In addition, we were aiming for an early discharge. We therefore wanted to choose an oral anticoagulant and selected a DOAC as the first-line treatment. The patient's DIC rapidly improved thereafter (Fig. 2). Neither bleeding nor thrombosis was seen. Moreover, since no worsening of the patient's quality of life was seen, treatments for both lung cancer and DIC could be continued safely on an outpatient basis. If no recovery of the thrombocytopenia that accompanied DIC had been seen in this patient, the chemotherapy for lung cancer, which was causing bone marrow suppression, could not have been continued. We therefore believe that successful treatment of DIC was highly significant - not only because it improved the patient's DIC, but also because it enabled the lung cancer treatment to be safely continued.

As mentioned above, we previously reported that rivaroxaban is effective for treating chronic DIC (5). However, this is the first report to show a case in which DIC, which had been uncontrolled under the administration of warfarin, dramatically improved after switching to rivaroxaban.

Warfarin exerts anticoagulation activity by reducing the 

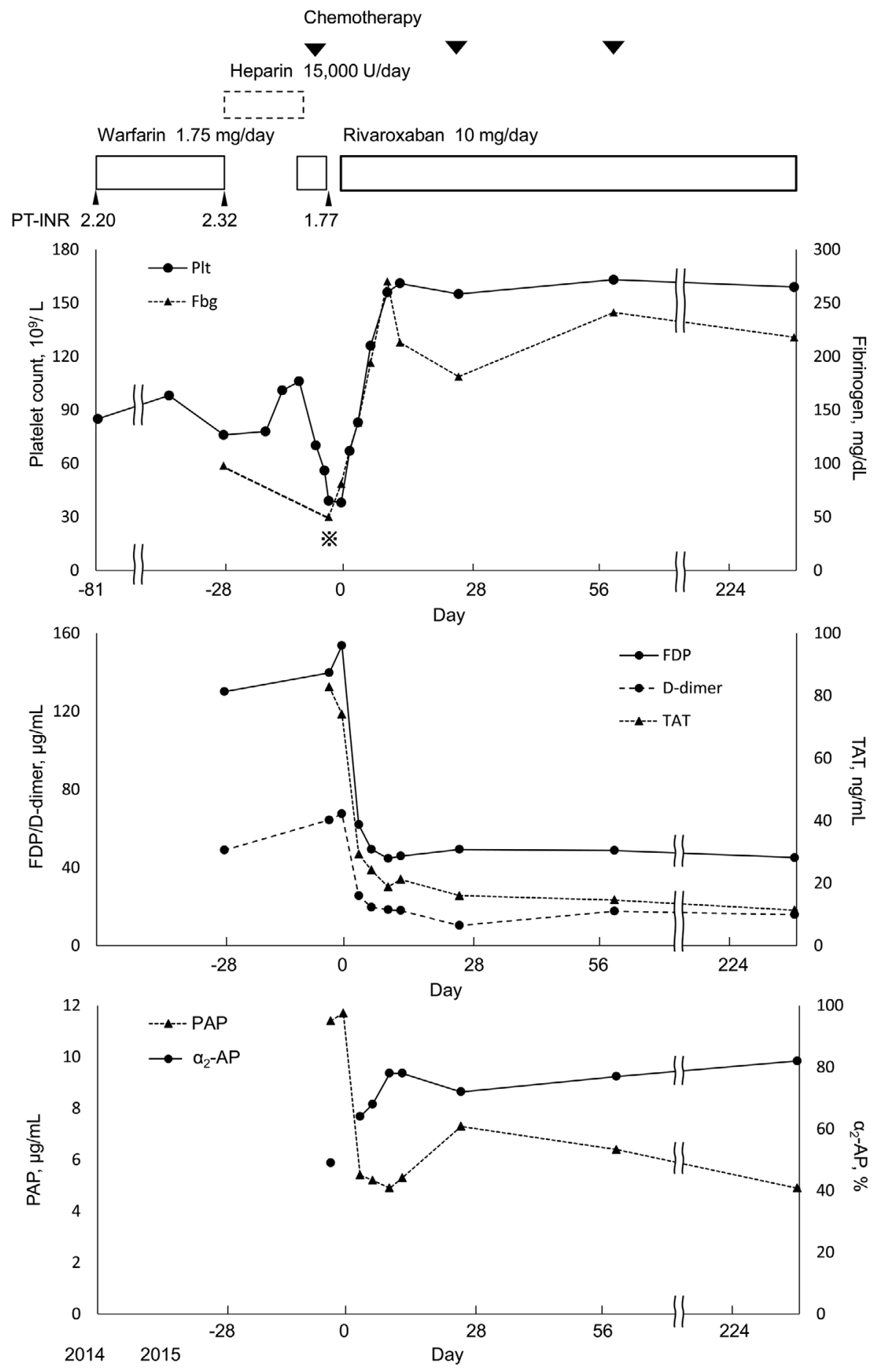

Figure 2. The clinical course of anticoagulant therapy and the changes in coagulation and fibrinolytic markers. Fbg<50 mg/dL. Day 0 indicates the day on which rivaroxaban treatment was initiated. Fbg: fibrinogen, FDP: fibrin/fibrinogen degradation products, TAT: thrombin antithrombin complex, PAP: plasmin- $\alpha_{2}$-antiplasmin complex, $\alpha_{2}$-AP: $\alpha_{2}$-antiplasmin

activity of vitamin K-dependent coagulation factors as substrates and proenzymes. Anticoagulation treatments using heparin and the like are effective against DIC. For that reason, warfarin, which is also an anticoagulant, was anticipated to be effective for DIC-despite the fact that it was not indicated for this condition. In fact, some reports have described the administration of warfarin in the treatment of DIC (6-10). Warfarin has an advantage in that it can be ad- ministered orally, which makes it easy to prescribe to outpatients. However, it is hard to ascertain whether warfarin really alleviated DIC in the above-mentioned reports, when they are evaluated from the current medical perspective. In addition, warfarin is associated with a drawback: the appetite loss caused by chemotherapy induces poor warfarin control.

In the present case, although warfarin could have been 
considered to be achieving adequate control based on the PT-INR, the decreased platelet count and the marked elevations of the FDP and D-dimer levels, which continued for a number of years, indicated that the patient's DIC was not being controlled. We responded to an exacerbation of DIC by discontinuing warfarin and switching to rivaroxaban, which resulted in the prompt alleviation of the patient's DIC, with a marked improvement in the platelet count and fibrinogen.

Our experience with this patient shows that the control of DIC requires the inhibition of the activated coagulation factors, such as thrombin and activated factor $\mathrm{X}$, rather than coagulation factors, which act as substrates. The present case is thus important in the sense that it shows that warfarin is ineffective for treating DIC, even in patients in whom DOAC treatment is effective.

Interestingly, the administration of rivaroxaban as an anticoagulation agent inhibited not only coagulation activation, but also fibrinolytic activation. The mechanisms underlying these dual effects are unclear. In addition, the use of rivaroxaban to treat DIC associated with underlying diseases other than aortic aneurysms has not been reported, and likewise, no reports have described the efficacy of DOACs other than rivaroxaban in the treatment of DIC. These issues need to be investigated further.

In summary, we reaffirmed that rivaroxaban was effective in treating DIC associated with an aortic aneurysm. At the same time, warfarin was observed to clearly be ineffective for treating DIC.

The authors state that they have no Conflict of Interest (COI).

\section{References}

1. Levi M, Ten Cate H. Disseminated intravascular coagulation. $\mathrm{N}$ Engl J Med 341: 586-592, 1999.

2. Takada A, Takada Y, Mori T, Sakaguchi S. Prevention of severe bleeding by tranexamic acid in a patient with disseminated intravascular coagulation. Thromb Res 58: 101-108, 1990.

3. Wada H, Matsumoto T, Yamashita Y. Diagnosis and treatment of disseminated intravascular coagulation (DIC) according to four DIC guidelines. J Intensive Care 2: 15, 2014.

4. Levi M, Toh CH, Thachil J, Watson HG. Guidelines for the diag- nosis and management of disseminated intravascular coagulation. British Committee for Standards in Haematology. Br J Haematol 145: 24-33, 2009.

5. Hayashi T, Nakagawa N, Kadohira Y, Morishita E, Asakura H. Rivaroxaban in a patient with disseminated intravascular coagulation associated with an aortic aneurysm: a case report. Ann Intern Med 161: 158-159, 2014.

6. Munter G, Hershko C. Increased warfarin sensitivity as an early manifestation of occult prostate cancer with chronic disseminated intravascular coagulation. Acta Haematol 105: 97-99, 2001.

7. Rubin RN, Colman RW. Disseminated intravascular coagulation. Approach to treatment. Drugs 44: 963-971, 1992.

8. Sack GH Jr, Levin J, Bell WR. Trousseau's syndrome and other manifestations of chronic disseminated coagulopathy in patients with neoplasms: clinical, pathophysiologic, and therapeutic features. Medicine 56: 1-37, 1977.

9. Fukuda N, Shimohakamada Y, Nakamori Y, et al. Thoracic aortic aneurysm with chronic disseminated intravascular coagulation treated successfully with orally administered camostat mesilate, warfarin and aspirin. Rinsho Ketsueki 43: 199-203, 2002 (in Japanese, Abstract in English).

10. Mori K, Suzuki S, Ishikawa M, Akutsu Y, Toyota T, Sakai H. A case of Kasabach-Merrit syndrome complicated with DIC treated effectively by long term oral administration of warfarin. Rinsho Ketsueki 36: 200-205, 1995 (in Japanese, Abstract in English).

11. Asakura $\mathrm{H}$. Classifying types of disseminated intravascular coagulation: clinical and animal models. J Intensive Care 2: 20, 2014.

12. Ontachi Y, Asakura H, Omote M, Yoshida T, Matsui O, Nakao S. Kasabach-Merritt syndrome associated with giant liver hemangioma: the effect of combined therapy with danaparoid sodium and tranexamic acid. Haematologica 90: e83-e85, 2005.

13. Ontachi Y, Asakura H, Arahata M, et al. Effect of combined therapy of danaparoid sodium and tranexamic acid on chronic disseminated intravascular coagulation associated with abdominal aortic aneurysm. Circ J 69: 1150-1153, 2005.

14. Iyama $\mathrm{S}$, Sato $\mathrm{T}$, Murase $\mathrm{K}$, et al. Intermittent administration of recombinant human soluble thrombomodulin successfully controlled chronic disseminated intravascular coagulation in a patient with dissecting aortic aneurysm on an outpatient basis. Blood Coagul Fibrinolysis 23: 548-550, 2012.

15. Kawano H, Hata T, Uda A, Maemura K. Use of rivaroxaban for the effective management of disseminated intravascular coagulation associated with abdominal aortic aneurysm. Intern Med 54: 2625-2628, 2015.

The Internal Medicine is an Open Access article distributed under the Creative Commons Attribution-NonCommercial-NoDerivatives 4.0 International License. To view the details of this license, please visit (https://creativecommons.org/licenses/ by-nc-nd/4.0/).

(C) 2017 The Japanese Society of Internal Medicine

Intern Med 56: 2913-2917, 2017 